\title{
Two cases of laparoscopic total colectomy with natural orifice specimen extraction and review of the literature
}

\author{
Ersin Gundogan, Aydin Aktas, Cuneyt Kayaalp, Fatih Gonultas, Fatih Sumer \\ Department of Surgery, Inonu University, Malatya, Turkey \\ Videosurgery Miniinv 2017; 12 (3): 291-296 \\ DOI: https://doi.org/10.5114/wiitm.2017.69227
}

\begin{abstract}
We present two cases of natural orifice specimen extraction (NOSE) after laparoscopic total colectomy and ileorectal anastomosis (TC-IRA), and we also review all of the previously reported cases. Our aim was to focus on patient selection for NOSE after TC-IRA. The PubMed and Google Scholar databases were scanned. Demographic features, surgical indications, and techniques were analyzed. Basic calculations were used for statistical analysis. A total of 13 cases were detected in addition to our 2 cases. All of the specimens were removed through the natural orifices successfully. No case required a diverting ileostomy. No patients were converted to open surgery or to conventional laparoscopy. Complications were reported in three patients. Transanal extractions were performed in 12 cases (10 colonic inertia, 2 polyposis), and transvaginal extractions were performed in 3 cases ( 2 malignancy, 1 colonic inertia). Both transanal and transvaginal specimen extractions after laparoscopic TC-IRA can be preferred. However, transanal extraction seems to be feasible in cases of TC for benign disease with a limited mesenteric-omental resection. If the indication is a malignancy requiring a mesenteric-omental resection, a transvaginal route should be preferred for a voluminous specimen.
\end{abstract}

Key words: natural orifice surgery, slow transit constipation, colorectal cancer, laparoscopic colorectal surgery, review, transrectal.

\section{Introduction}

Minimally invasive colorectal surgery began in the early 1990s. It has gained popularity due to its advantages over open surgery, such as lower morbidity rates and rapid patient convalescence. Laparoscopic total colectomy and ileorectal anastomosis (TC-IRA) was a relatively less applied colorectal procedure. It was challenging because of the requirement to work laparoscopically in all four quadrants of the abdomen. During laparoscopic TC-IRA, an abdominal mini-laparotomy was always required for specimen extraction and anastomosis. This mini-laparotomy could increase wound-related morbidities and have a negative influence on postoperative quality of life. To avoid the mini-laparotomy, natural orifice spec- imen extraction (NOSE) after laparoscopic TC-IRA has been recently described [1-6]. However, there is no consensus as to which patients are suitable for transvaginal or transanal specimen extractions after laparoscopic TC-IRA.

The aim of this study with two new cases was to examine all the cases in the literature and investigate the appropriate NOSE route after laparoscopic TC-IRA.

\section{Case reports}

\section{Surgical technique}

Surgery was performed in the French position. An umbilical $12 \mathrm{~mm}$ trocar was placed with the open

\footnotetext{
Address for correspondence

Prof. Cuneyt Kayaalp, Department of Surgery, Inonu University, 44315 Malatya, Turkey, phone: +90 4223774001 ,

e-mail: cuneytkayaalp@hotmail.com
} 
technique. Right and left lower abdominal $12 \mathrm{~mm}$ trocars and both lumbar $5 \mathrm{~mm}$ trocars were placed. First, the sigmoid and descending colons were dissected from the lateral abdominal wall and the mesocolon was divided close to the mesenteric border of the colon via LigaSure $10 \mathrm{~mm}$ (Medtronic, US). The splenic flexure of the colon was mobilized and divided. The greater omentum was not removed and the remaining colon segments (transverse, hepatic flexure, ascendant, and cecum) were all separated from the mesocolon in the same way. The recto-sigmoid junction and distal ileum were transected separately using $60 \mathrm{~mm}$ linear endoscopic staplers (Blue cartridge, Ethicon, Johnson \& Johnson, US). The recto-sigmoid stump was opened with scissors and the free abdominal specimen was taken out of the anus by an ovarian clamp (Photos 1, 2).

\section{Case 1}

A 63-year old man was admitted with a positive fecal occult test. He had type 2 diabetes mellitus and a previous open prostatectomy for cancer 7 years ago. Physical examination revealed a lower midline incision scar. Colonoscopy demonstrated normal rectum and multiple colonic polyps that could not be removed endoscopically. The patient was diagnosed with attenuated familial adenomatous polyposis. Endoscopic biopsy from one of the polyps revealed tubulovillous adenoma with high-grade dysplasia. An abdominal ultrasound detected cholelithiasis. The NOSE after laparoscopic TC-IRA combined with cholecystectomy was planned after informed consent from the patient.

The port sites and the position of the patient were as described above. Cholecystectomy was com-

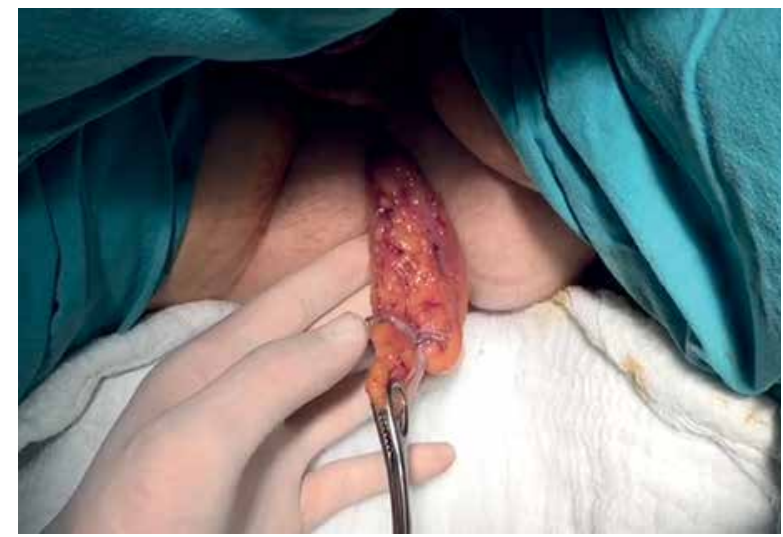

Photo 1. Transanal extraction of the specimen pleted and the gall bladder was removed through the $12 \mathrm{~mm}$ trocar site. Laparoscopic TC and NOSE were done as described in the surgical technique portion. The rectal stump was re-closed with a $60 \mathrm{~mm}$ linear stapler. The IRA was accomplished side-to-side using a $60 \mathrm{~mm}$ linear stapler, and the common orifice of the stapler opening was closed by intracorporeal suturing. No diverting ileostomy was done and one pelvic drain was placed. Oral fluids were started on day one. Postoperative analgesics were required six times (three dosages of paracetamol and three dosages of non-steroid anti-inflammatory analgesics). Defecations started on day one (four per day) and continued at 2-3 times per day. The postoperative course was uneventful except for pneumonia, which was treated with intravenous antibiotics. Histopathological examination revealed tubulovillous adenomas, and one of them had high-grade dysplasia. On the $30^{\text {th }}$ day after surgery he was readmitted for oral intolerance in the absence of fever or pain. He had raised white blood cell counts. Computed tomography diagnosed a pelvic abscess, and it was treated successfully with percutaneous drainage and antibiotics. He was well on the $45^{\text {th }}$ day after surgery.

\section{Case 2}

A 42-year-old woman was referred to us for attenuated polyposis coli. Colonoscopy revealed a normal rectum but with multiple polyps in the colon. She had previous surgery for a lumbar disc hernia. Laparoscopic TC and NOSE were done as described as above. The IRA was done with a circular stapler. The anvil of the stapler was introduced into the abdomen through the open rectal stump and then the rectal stump was closed with a laparoscopic linear stapler.

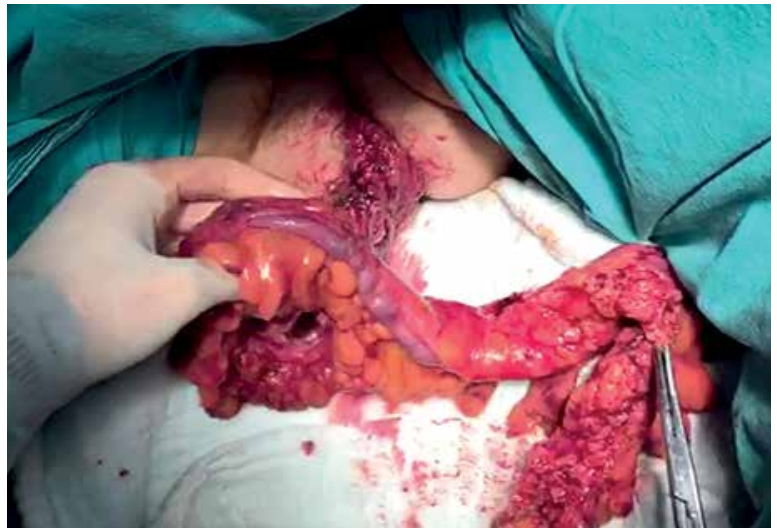

Photo 2. Transanal extraction of the whole colon 
The anvil was placed into the ileum intracorporeally and the IRA was accomplished end-to-side. No diverting ileostomy was done and one pelvic drain was placed. Oral fluids were started on day one. Postoperative analgesics were required 10 times (four dosages of pethidine $\mathrm{HCl}$ and six dosages of paracetamol). In the postoperative period, there was an ileus that required cessation of oral intake but it was resolved with medical treatment. Histopathological examination confirmed the adenomatous polyposis and she was well after 5 weeks of surgery.

\section{Review of the literature}

Electronic searches of the PubMed/Medline and Google Scholar databases were carried out on 27 October 2016 using the key words (transvaginal OR transanal OR transrectal) AND ((colon OR colonic) AND (resection OR resections) OR colectomy) AND (total OR subtotal). All of the patients who had laparoscopic total colectomy and transanal or transvaginal specimen extractions were included. Patients were included irrespective of age, sex, region, race, or obesity. Patients who underwent laparoscopic total proctocolectomy or segmented colectomies were excluded. No restrictions were made on language, country, or journal. The "related articles" links of the databases and the references of all of the selected relevant articles were cross-checked. Two reviewers (AA, CK) assessed the list of titles and/or abstracts of the scanned articles. If the articles were obviously irrelevant to the aim of this analysis they were excluded. Articles that met our inclusion criteria were obtained in their full text form for assessment. The reasons for excluding studies were recorded in the flow chart (Figure 1). Any disagreements during the study selection and analysis were solved by reviewer discussion and a consensus was obtained. Data for publication year, country, patient gender, age, surgical indication, natural orifice for extraction, duration of surgery, blood loss, type of ileo rectal anastomosis, morbidity, length of hospital stay, and follow-up time were recorded. A computer program including a spreadsheet was used for records (Excel). Basic descriptive statistics were used for analysis.

\section{Results}

We found a total of 13 cases in six studies and we added our cases as the $14^{\text {th }}$ and the $15^{\text {th }}$ patients (Tables I, II). Three prior studies were from the Unit-

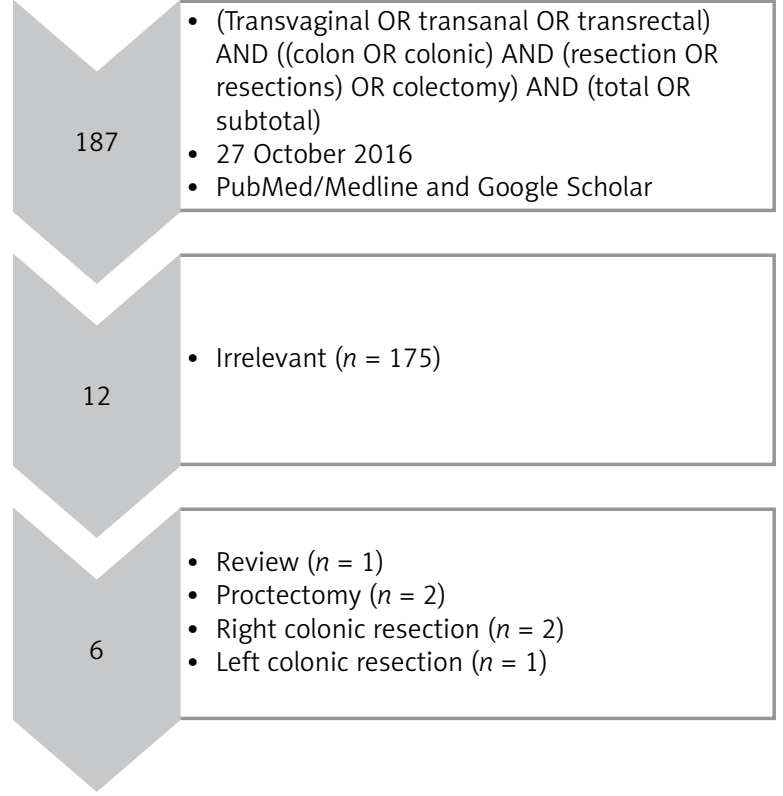

Figure 1. Flowchart of the systematic review

ed States and the remaining studies were from China, Taiwan, and Panama. There was no report from Europe or South America where NOSE was invented and expanded $[7,8]$. The mean age of the available patients was $50 \pm 16$ and gender was reported only in 6 patients ( 5 females and 1 male). The indications for TC-IRA were colonic inertia $(n=11)$, hereditary nonpolyposis colorectal cancer $(n=1)$, attenuated familial adenomatous polyposis $(n=2)$, and sigmoid colon cancer $(n=1)$. The number of trocars ranged between 4 and 6 and all of the anastomoses were done intracorporeally. Anastomosis was created usually with a circular stapler, with the exception of one side-to-side case with linear staplers (our first case). The TC-IRA was performed in all cases without any conversion to open surgery or to conventional laparoscopic surgery. No patients required a diverting ileostomy. Twelve surgical specimens were extracted through the anus and three specimens through the vagina. Two malignant colon specimens were removed from the vagina and the 13 benign specimens were all removed mainly from the anus, but one from the vagina. The specimens were removed from the abdomen by a clamp or extractions and were assisted with an endoscope. The mean operating time was $297 \pm 100 \mathrm{~min}(180-540 \mathrm{~min})$ and the mean blood loss was $116 \pm 94 \mathrm{ml}(10-400 \mathrm{ml})$. The mean length of hospital stay was $5.3 \pm 2.7$ days (range: 2-9 days). Complications were reported in 3 patients. 
Table I. Literature review of total colectomy with ileoractal anastomosis and natural orifice specimen extraction: patients' demographics and surgical indications

\begin{tabular}{|c|c|c|c|c|c|c|}
\hline Author & Year & Country & No. & Gender & Age & Indication \\
\hline Fan et al. & 2016 & Taiwan & 1 & NA & NA & Constipation \\
\hline Gong et al. & 2014 & China & 8 & NA & NA & Constipation \\
\hline Awad et al. & 2014 & USA & 1 & Female & NA & Sigmoid cancer + polyposis coli \\
\hline Awad et al. & 2012 & USA & 1 & Female & 27 & Colonic inertia \\
\hline Dozois et al. & 2008 & USA & 1 & Female & 53 & Hereditary non-polyposis coli \\
\hline Rodríguez et al. & 2013 & Panama & 1 & Female & 65 & Constipation \\
\hline Gundogan et al. & 2016 & Turkey & 1 & Male & 63 & Attenuated familial adenomatous polyposis \\
\hline Gundogan et al. & 2016 & Turkey & 1 & Female & 42 & Attenuated familial adenomatous polyposis \\
\hline
\end{tabular}

Table II. Literature review of total colectomy with ileoractal anastomosis and natural orifice specimen extraction: surgical details and postoperative courses

\begin{tabular}{|c|c|c|c|c|c|c|c|}
\hline Author & $\begin{array}{l}\text { No. of } \\
\text { trocars }\end{array}$ & Anastomosis & $\begin{array}{l}\text { Specimen } \\
\text { extraction }\end{array}$ & $\begin{array}{l}\text { Operation } \\
\text { time [min] }\end{array}$ & $\begin{array}{l}\text { Blood loss } \\
{[\mathrm{ml}]}\end{array}$ & $\begin{array}{l}\text { Postoperative } \\
\text { complication }\end{array}$ & LOS \\
\hline Fan et al. & 5 & End-to-side & Transanal & NA & NA & NA & NA \\
\hline Gong et al. & NA & NA & Transanal & $287.6 \pm 21.5$ & $109.7 \pm 41.1$ & NA & NA \\
\hline Awad et al. & 6 & End-to-side & Transvaginal & 210 & 20 & None & 2 \\
\hline Awad et al. & 5 & End-to-side & Transanal & 180 & 10 & NA & 4 \\
\hline Dozois et al. & 4 & End-to-end & Transvaginal & 455 & 400 & Ileus & 7 \\
\hline Rodríguez et al. & NA & NA & Transvaginal & NA & NA & None & 3 \\
\hline Gundogan et al. & 5 & Side-to-side & Transanal & 540 & 150 & Pneumonia \& SSI & 9 \\
\hline Gundogan et al. & 5 & End-to-side & Transanal & 180 & 50 & Ileus & 7 \\
\hline
\end{tabular}

$N A$ - not available, SSI - surgical site infection, LOS - length of stay.

\section{Discussion}

We concluded that both transanal and transvaginal specimen extractions after laparoscopic TC-IRA are feasible. However, transanal extractions seemed to be preferred in cases of TC for a benign disease (e.g. colonic inertia) with a limited mesenteric-omental resection (Figure 2). If the indication was a malignancy that required a mesenteric-omental resection, the transvaginal route should be preferred for a voluminous specimen (Figure 3). Transanal specimen extraction can be performed for smaller volume materials due to distal bowel diameter and anal sphincters. Because the vagina is more expandable than the bowel and has no sphincters, the transvaginal route allows larger masses for extraction. In a male patient with a voluminous TC specimen (i.e. including the total mesocolon), specimen removal through a suprapubic incision should be preferred.
The indications of TC-IRA were limited to: (i) some colon cancers (hereditary nonpolyposis colorectal cancer, synchronous cancers, and left-sided acute colonic obstructive cancers), (ii) some pre-malignant diseases (familial adenomatous polyposis without rectal involvement or attenuated form), (iii) some inflammatory bowel diseases (especially in young women with mild rectal disease, no dysplasia, and normal rectal compliance) or (iv) slow transit constipation. Except for emergency left-sided colonic cancer obstructions, laparoscopic TC-IRA seems to be feasible for all of these indications.

When previous publications were examined, Awad used transvaginal extraction instead of transanal possibly due to the bulky omental and mesenteric tissue in a patient with laparoscopic TC for colon cancer [2]. The same author preferred the transanal route in a patient with colonic inertia [3]. Interestingly, Rodriguez-Zentner et al. opted 


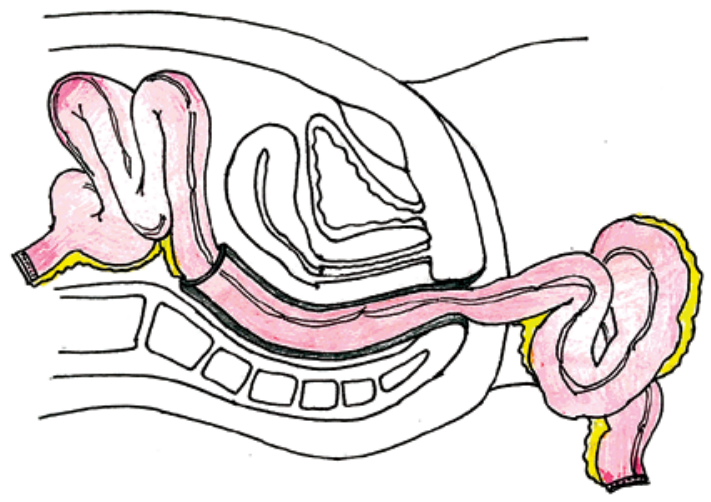

Figure 2. Transanal extraction seemed to be feasible in cases of total colectomy for benign disease with a limited mesenteric-omental resection

to remove the specimen in a patient with colonic inertia through the vagina [5]. In our opinion, the transanal route should be preferred instead of transvaginal for benign colonic pathology that does not necessitate mesenteric resection. By this approach, an additional trauma to innocent organs can be avoided.

It is well known that laparoscopic surgery reduces both general complications and wound-related complications when compared to open surgery [9]. Despite the advantages of laparoscopic surgery, wound-related complications after laparoscopic colorectal surgery still have significant rates. In a recent nationally based study on TC-IRA [8], the rates of deep and superficial surgical site complications were $10.1 \%$ in open surgery and $8 \%$ in laparoscopic surgery. NOSE is a technique that has the potential for fewer wound-related early and late complications than laparoscopic surgery $[10,11]$. In this review, we did not find any wound-related complications after laparoscopic TC-IRA when combined with NOSE. Decreased wound complications may prevent the delay of postoperative adjuvant chemo-radio therapy in malignant patients. Decreased wound complications will also decrease post-operative pain and provide faster convalescence. It is obvious that NOSE will be more advantageous in terms of cosmetics. Despite these benefits, NOSE still has some risks such as intra-abdominal organ injuries, sphincter damage due to anal stretching, or dyspareunia. Fortunately, these complications are very rare [12].

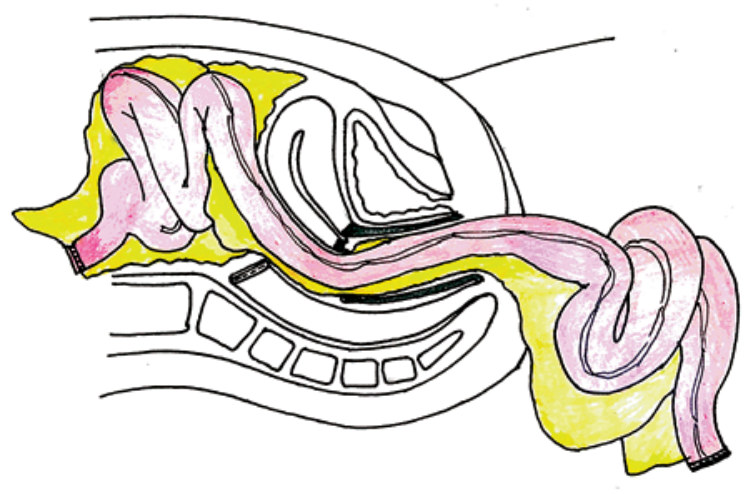

Figure 3. If the indication of total colectomy was a malignancy that required a mesenteric-omental resection, a transvaginal route should be preferred for a voluminous specimen

The limitations of this review were the number of patients, lack of long-term outcomes and comparative studies, and lack of confidence in the use of malignant diseases. The removal of a specimen from a natural orifice may not be appropriate for patients with vaginal anomalies, patients with very large specimens, anal stenosis, or in patients unable to position due to a lower extremity deformity. In addition, cultural sensitivity regarding the use of the vagina for surgical specimen extraction can be a problem.

\section{Conclusions}

Laparoscopic TC-IRA is an accepted procedure and when compared to open surgery has the advantage of lower complication rates and quicker recovery. After laparoscopic TC-IRA, either the anus or the vagina can be used as the specimen extraction site. This may potentiate the advantages of laparoscopic surgery.

\section{Conflict of interest}

The authors declare no conflict of interest.

\section{References}

1. Dozois EJ, Larson DW, Dowdy SC, et al. Transvaginal colonic extraction following combined hysterectomy and laparoscopic total colectomy: a natural orifice approach. Tech Coloproctol 2008; 12: 251-4.

2. Awad Z. Laparoscopic total colectomy with transvaginal extraction of the colon and ileorectal anastomosis. Ann Surg Oncol 2014; 21: 3029. 
3. Awad ZT. Laparoscopic subtotal colectomy with transrectal extraction of the colon and ileorectal anastomosis. Surg Endosc 2012; 26: 869-71.

4. Fan WC, Huang CC, Sung A, Hsieh JS. Laparoscopic total colectomy with transrectal specimen extraction and intraabdominal ileorectal anastomosis for slow-transit constipation (with vid eo). J Visc Surg 2016; 153: 309-10.

5. Rodríguez-Zentner H, Juárez H, Ríos J, et al. Total colectomy with transvaginal specimen extraction due to colonic inertia. Rev Gastroenterol Mex 2014; 79: 153-4.

6. Gong W, Yang X, Song C, et al. Laparoscopy-assisted subtotal colectomy with transanal specimen extraction for slow transit constipation. Zhonghua Wei Chang Wai Ke Za Zhi 2014; 17: 796-8.

7. Zorrón R, Filgueiras M, Maggioni LC, et al. NOTES. Transvaginal cholecystectomy: report of the first case. Surg Innov 2007; 14 279-83.

8. Lehmann KS, Zornig C, Arlt G, et al. Natural orifice transluminal endoscopic surgery in Germany: data from the German NOTES registry. Chirurg 2015; 86: 577-86.

9. Onder A, Benlice C, Church J, et al. Short-term outcomes of laparoscopic versus open total colectomy with ileorectal anastomosis: a case-matched analysis from a nationwide database. Tech Coloproctol 2016; 20: 767-773.

10. Ma B, Huang XZ, Gao P, et al. Laparoscopic resection with natural orifice specimen extraction versus conventional laparoscopy for colorectal disease: a meta-analysis. Int J Colorectal Dis 2015; 30: 1479-88.

11. Kayaalp C, Yagci MA, Soyer V. Laparoscopic and natural orifice transluminal restorative proctocolectomy: no abdominal incision for specimen extraction or ileostomy. Videosurgery Miniinv 2016; 11: 115-20.

12. Wood SG, Panait L, Duffy AJ, et al. Complications of transvaginal natural orifice transluminal endoscopic surgery: a series of 102 patients. Ann Surg 2014; 259: 744-9.

Received: 20.11.2016, accepted: 7.05.2017. 